\title{
Classical Solution for Ghost D-Branes in String Field Theory
}

\author{
Syoji Zeze \\ Yokote Seiryo Gakuin High School, 147-1 Maeda, Osawa, Yokote 013-0041, Japan \\ Correspondence should be addressed to Syoji Zeze; ztaro21@gmail.com
}

Received 1 July 2017; Accepted 2 October 2017; Published 29 October 2017

Academic Editor: Elias C. Vagenas

Copyright (c) 2017 Syoji Zeze. This is an open access article distributed under the Creative Commons Attribution License, which permits unrestricted use, distribution, and reproduction in any medium, provided the original work is properly cited. The publication of this article was funded by $\mathrm{SCOAP}^{3}$.

Ghost D-branes have been proposed as objects that cancel the effects of D-branes. We construct a classical solution which represents an arbitrary number of D-branes and ghost D-branes in the context of open string field theory. Cancellation of BRST cohomology between $\mathrm{D}$-branes and ghost $\mathrm{D}$-branes is shown.

\section{Introduction}

Recent developments in open string field theory (OSFT) [1] have proved that it is able to describe wide variety of open string backgrounds. A prominent example is the solution found by Erler and Maccaferri [2] which covers wide range of open string backgrounds such as marginal deformation, Dbrane lump, and multiple D-branes. More recently, Kojita et al. have incorporated topological defects between boundary conformal field theories (BCFTs) [3] into OSFT context [4]. A common belief behind those developments is that OSFT governs the landscape of (hopefully all possible) BCFTs. Studies on explicit background are still important in order to understand the nature of the landscape.

In this paper, we are interested in ghost D-branes, which are rather different from D-branes in conventional BCFTs. Originally, ghost $\mathrm{D}$-branes (gD-branes) were introduced by Okuda and Takayanagi as objects that cancel the effect of D-branes [5] and studied subsequently by some authors [6, 7] (the author became aware of works [8-13] which also deal with branes with negative tension. We thank S. E. Parkhomenko for correspondence). It is characterized by a boundary state with opposite sign:

$$
|B(\mathrm{gD})\rangle=|B(\mathrm{D})\rangle .
$$

Then, the amplitude for a closed string propagating between $\mathrm{D}$ - and gD-branes is given by $\langle B(\mathrm{gD})|\Delta| B(\mathrm{D})\rangle=$ $-\langle B(D)|\Delta| B(D)\rangle$, where $\Delta$ is an inverse of the closed string propagator. Obviously, this has a sign opposite to the amplitude with two D-branes. In open string channel, this amplitude can be interpreted as one-loop partition functions of open strings stretched between branes. The negative sign of $\mathrm{D}$-gD partition function can be attributed to fermionic path integral. Thus, the field content on a coincident $\mathrm{D}-\mathrm{gD}$ pair should be

$$
\left(\begin{array}{cc}
\varphi_{1} & \psi \\
\psi^{\dagger} & \varphi_{2}
\end{array}\right)
$$

where $\varphi_{1}$ and $\varphi_{2}$ are bosonic, while $\psi$ and $\psi^{\dagger}$ are fermionic. Extension to $N$ D-branes and $M$ gD-branes gives rise to $U(N \mid M)$ or $O S p(N \mid M)$ matrix with similar structure as (2). The authors of [5] showed that D- and gD-branes cancel each other in the partition function of supermatrix model defined by (2). With that result, they claimed that a $\mathrm{gD}$ brane cancels D-brane completely; therefore, a $\mathrm{D}-\mathrm{gD}$ pair is equivalent to the tachyon (or closed) string vacuum. This means that all physical observables cancel between $\mathrm{D}$ - and gD-branes.

Subsequently, several authors encountered gD-branes in the efforts of constructing classical solution for multiple D-branes in OSFT [14-19] $([20,21]$ also rediscovered gDbranes as boundary states). In this context, a gD-brane is given by a classical solution with negative tension. It has not drawn much attention because of its peculiar nature. However, it is worthwhile to mention that single gD-brane was found to be regular solution in all existing literature. In particular, the gD-brane solution discovered by Masuda, 
Noumi, and Takahashi (MNT) [17] passed thorough most stringent consistency checks ever known. On the other hand, a solution for multiple D-branes in universal space, which was main aim of the authors of [14-19], turned out to fail such consistency checks unless quite subtle regularization (for example, phantom term) is assumed. Therefore, it is worth investigating gD-branes seriously in OSFT context.

In this paper, we will ask two questions about the nature of gD-branes. First, we will ask whether they are physical degrees of freedom. The negative tension of $\mathrm{gD}$-branes implies inconsistency of bosonic OSFT. Therefore, it is important to ask whether there exists logic to exclude gD-brane from the spectrum. Unfortunately, we will not have conclusive answer to this question. We will argue with regard to possible solutions to this problem in terms of gauge fixing.

Second, we will ask how gD-branes in OSFT differ from the original picture by Okuda and Takayanagi. A crucial difference between them is seen in a way of cancellation between $\mathrm{D}$ - and gD-branes. In the original picture, the cancellation completely holds at quantum level since partition function becomes trivial for $\mathrm{D}$-gD pairs. One the other hand, the cancellation in OSFT was confirmed only for physical observables. To close the gap between two, we will propose another criterion for the cancellation. Namely, it is cancellation of BRST cohomology. It will be shown that the BRST cohomology of D-gD pairs vanishes with suitable choice of the string field.

Before going into details, we briefly sketch main results of our paper. MNT gD-brane [17] is simply given by a sum of two "tachyons":

$$
\Psi_{\mathrm{MNT}}=\Psi_{F}+\Psi_{H}
$$

where $\Psi_{F}$ and $\Psi_{H}$ are Okawa-type analytic solutions [22] that are orthogonal with each other:

$$
\Psi_{F} \Psi_{H}=\Psi_{H} \Psi_{F}=0
$$

In order to prove D-gD cancellation of cohomology, we fix $\Psi_{F}$ to be tachyon and switch to the vacuum defined by

$$
Q_{F}=Q_{B}+\left\{\Psi_{F}, *\right\} .
$$

Then, a solution for a $\mathrm{D}-\mathrm{gD}$ pair is identified as

$$
\Psi_{\mathrm{D}+\mathrm{gD}}=-\Psi_{F}+\Psi_{H}
$$

BRST cohomology around this solution vanishes as we will see, since

$$
Q_{\mathrm{D}+\mathrm{gD}}=Q_{F}+\left\{\Psi_{\mathrm{D}+\mathrm{gD}}, *\right\}=Q_{B}+\left\{\Psi_{H}, *\right\}
$$

just corresponds to a kinetic operator for "another" tachyon. Thus, we have shown that a D-gD pair $\Psi_{\mathrm{D}+\mathrm{gD}}$ has no cohomology; therefore, physical excitations cancel out. We also construct a solution with an arbitrary number of $\mathrm{D}$ - and gD-branes:

$$
\Psi_{N, M}=-\sum_{a=1}^{N} \Sigma_{k_{a}} \Psi_{F} \bar{\Sigma}_{k_{a}}+\sum_{a=1}^{M} \Sigma_{l_{a}} \Psi_{H} \bar{\Sigma}_{l_{a}}
$$

where $\Sigma_{k_{a}}$ and $\bar{\Sigma}_{k_{a}}$ are the modified BCC projectors introduced in [2]. It will be shown that the cohomology does not always cancel but cancels in a subset of whole string fields.

This paper is organized as follows. In Section 2, after a brief review of the MNT solution [17], we will introduce a set of classical solutions which describes the D-gD system. Cancellation between $\mathrm{D}$ - and gD-branes will be confirmed for gauge invariant observables and BRST cohomology. In Section 3, we will extend this result to multiple branes. We will conclude in Section 4 with some discussions.

\section{MNT Ghost D-Brane}

2.1. Solutions. We begin with a study of the ghost Dbrane solution discovered by Masuda, Noumi, and Takahashi (MNT) [17]. As explained in Introduction, it is simply a sum of formal solutions [22] of equation of motion around the perturbative vacuum:

$$
\Phi_{\mathrm{MNT}}=\Psi_{F}+\Psi_{H}
$$

where

$$
\begin{aligned}
\Psi_{F} & =F c \frac{K}{1-F^{2}} B c F, \\
\Psi_{H} & =H c \frac{K}{1-H^{2}} B c H,
\end{aligned}
$$

where $K, B$, and $c$ are elements of the $K B c$ algebra and $F$ and $H$ are functions of $K$. Since the equation of motion is quadratic, a sum of two Okawa solutions never becomes a solution unless their product vanishes; that is,

$$
\Psi_{F} \Psi_{H}=\Psi_{H} \Psi_{F}=0
$$

We will refer to this relation as orthogonality. Okawa solutions in (10) become orthogonal when $F H=1$ holds since the product $c^{2}$ banishes. It is also required that both $\Psi_{F}$ and $\Psi_{H}=$ $\Psi_{F^{-1}}$ derive the expected values of the classical action (in this paper, normalized to be -1 ) and vanishing cohomologies. In addition, MNT imposed a consistency condition for the boundary state [23] to the solution. They showed an explicit choice

$$
\begin{gathered}
F=\sqrt{\frac{1-p K}{1-q K}}, \\
H=\sqrt{\frac{1-q K}{1-p K}},
\end{gathered}
$$

where $p$ and $q$ are positive numbers, fulfilling all requirements. With this choice, they claimed that solution (9) should be identified with a gD-brane. In order to confirm their claim, it is convenient to switch to the tachyon vacuum. We will fix $\Psi_{F}$ to be the "reference" tachyon vacuum (as is clear from (12), there is no essential difference between $F$ and $H$ since they exchange under $p \leftrightarrow q$. $\Psi_{F}$ and $\Psi_{H}$ are gauge equivalent since they derive same physical observables and therefore belong 
to the same class of analytic solutions [17]) so that OSFT is described by an action

$$
S_{F}[\Psi]=\operatorname{Tr}\left[\frac{1}{2} \Psi Q_{F} \Psi+\frac{1}{3} \Psi^{3}\right],
$$

where $Q_{F}=Q_{B}+\left\{\Psi_{F}, *\right\}$. Then, equation of motion of the action (13) is $Q_{F} \Psi+\Psi^{2}=0$ and we find four solutions composed of $\Psi_{F}$ and $\Psi_{H}$ :

$$
\begin{aligned}
\Psi_{\mathrm{D}} & =-\Psi_{F}, \\
\Psi_{\mathrm{TV}} & =0, \\
\Psi_{\mathrm{D}+\mathrm{gD}} & =-\Psi_{F}+\Psi_{H}, \\
\Psi_{\mathrm{gD}} & =\Psi_{H} .
\end{aligned}
$$

It is straightforward to confirm that all of them satisfy the equation of motion. We identify $\Psi_{\mathrm{D}}, \Psi_{\mathrm{TV}}, \Psi_{\mathrm{D}+\mathrm{gD}}$, and $\Psi_{\mathrm{gD}}$ as D-brane, tachyon vacuum, D-gD pair, and gD-brane, respectively. An evidence of this identification comes from values of tension: 1 for $\mathrm{D}$-brane, 0 for the tachyon vacuum and $\mathrm{D}-\mathrm{gD}$ pair, and -1 for gD-brane.

2.2. Gauge Invariant Observables. In this section, we will evaluate three gauge invariant observables for a $\mathrm{D}-\mathrm{gD}$ pair: classical action, gauge invariant overlap, and boundary state. Let $\mathcal{O}(\Psi)$ be a gauge invariant observable of interest. The cancellation of a physical observable for the $\mathrm{D}-\mathrm{gD}$ pair is represented by

$$
\mathcal{O}\left(\Psi_{\mathrm{D}+\mathrm{gD}}\right)=0 .
$$

The first observable is the brane tension, which is easily evaluated as follows:

$$
\begin{aligned}
S_{F}\left[\Psi_{\mathrm{D}+\mathrm{gD}}\right] & =S_{F}\left[\Psi_{\mathrm{D}}\right]+S_{F}\left[\Psi_{\mathrm{gD}}\right] \\
& =1+(-1) \\
& =0
\end{aligned}
$$

where we have used orthogonality in the first line.

The second is the gauge invariant overlap for closed string $[24,25]$. Evaluation proceeds straightforwardly as

$$
\begin{aligned}
\left\langle I|\mathscr{V}(i)| \Psi_{\mathrm{D}+\mathrm{gD}}\right\rangle & =\left\langle I|\mathscr{V}(i)| \Psi_{\mathrm{D}}\right\rangle+\left\langle I|\mathscr{V}(i)| \Psi_{\mathrm{gD}}\right\rangle \\
& =-\left\langle I|\mathscr{V}(i)| \Psi_{F}\right\rangle+\left\langle I|\mathscr{V}(i)| \Psi_{H}\right\rangle \\
& =0
\end{aligned}
$$

where $\mathscr{V}$ is a closed string vertex operator and $\langle I|$ is the identity string field. In third line, we used the fact that the values of the overlap for $\Psi_{F}$ and $\Psi_{H}$ are identical.

The third is the gauge invariant boundary state [26]. We will show the cancellation

$$
\left|B\left(\Psi_{\mathrm{D}+\mathrm{gD}}\right)\right\rangle=0,
$$

where $|B(\Psi)\rangle$ is a boundary state specified by an OSFT solution $\Psi$. As a first step, we will confirm that (21) holds for any physical state:

$$
\left\langle\mathscr{V}\left|c_{0}^{-}\right| B\left(\Psi_{\mathrm{D}+\mathrm{gD}}\right)\right\rangle=0
$$

The left hand side of the above equation is the correlation function which appears in well-known Ellwood conjecture (we use the formula presented in [26] rather than original one. We also omit $4 \pi i$ factor in right hand side of (23)) [24]:

$$
\left\langle\mathscr{V}\left|c_{0}^{-}\right| B(\Psi)\right\rangle=\langle I|\mathscr{V}(i)| \Psi\rangle-\left\langle I|\mathscr{V}(i)| \Psi_{\mathrm{TV}}\right\rangle,
$$

where all string fields should be classical solutions defined around the perturbative vacuum rather than the tachyon vacuum. Then, by introducing a shift $+\Psi_{F}$ to (16) and (15), a $\mathrm{D}-\mathrm{gD}$ pair and the tachyon vacuum are represented by $\Psi_{\mathrm{D}+\mathrm{gD}}=\Psi_{H}$ and $\Psi_{\mathrm{TV}}=\Psi_{F}$, respectively. Then we have

$$
\begin{aligned}
\left\langle\mathscr{V}\left|c_{0}^{-}\right| B\left(\Psi_{\mathrm{D}+\mathrm{gD}}\right)\right\rangle & =\left\langle I|\mathscr{V}(i)| \Psi_{H}\right\rangle-\left\langle I|\mathscr{V}(i)| \Psi_{F}\right\rangle \\
& =0,
\end{aligned}
$$

as expected. Note that we have used the result of (20) in the second line.

The cancellation can be extended further to arbitrary closed string states. Following Kudrna et al., we uplift string field to the auxiliary CFT [26]. Then, as explained in [26], the Ellwood formula (23) essentially contains all information of closed string state and therefore can be extended to arbitrary closed string state. Therefore, we have

$$
\left|B\left(\Psi_{\mathrm{D}+\mathrm{gD}}\right)\right\rangle=0,
$$

which can be interpreted as cancellation of boundary state (MNT confirmed the cancellation of boundary state in rather different way. They used KOZ boundary state [23] which is neither linear nor gauge invariant).

2.3. Cohomology. As explained in Introduction, it is easy to find empty cohomology of a D-gD pair. Namely, we evaluate the kinetic operator as

$$
\begin{aligned}
Q_{\mathrm{D}+\mathrm{gD}} & =Q_{F}+\left\{\Psi_{\mathrm{D}+\mathrm{gD}}, *\right\} \\
& =Q_{B}+\left\{\Psi_{F}, *\right\}+\left\{-\Psi_{F}+\Psi_{H}, *\right\} \\
& =Q_{B}+\left\{\Psi_{H}, *\right\}=Q_{H} .
\end{aligned}
$$

This is nothing but a BRST charge shifted by "another" tachyon vacuum specified $\Psi_{H}$ and therefore has a nontrivial homotopy operator [27]

$$
A=\frac{1-H^{2}}{K} B,
$$

and of course, its cohomology vanishes. This means that there are no physical excitations around a D-gD pair even at quantum level. An OSFT around a D-gD pair is completely equivalent to that around the tachyon vacuum since their cohomologies are identical. Thus, our result validates the original statement of [5]; a system with a pair of D-brane and ghost D-brane located at the same location is physically equivalent to the closed string vacuum in the context of OSFT. 
2.4. Projections. Our construction of D-gD system is largely owing to the orthogonality between $\Psi_{F}$ and $\Psi_{H}$. The identity $\left\{\Psi_{F}, \Psi_{H}\right\}=0$ means that $\Psi_{H}$ belongs to the kernel of the background shift generated by $\left\{\Psi_{F}, *\right\}$. It can be understood that such string fields are not limited to $\Psi_{H}$ but fill large part of the string fields. A crucial observation is that both $\Psi_{F}$ and $\Psi_{H}$ are projected string fields:

$$
\begin{gathered}
\Psi_{F}=p_{1} \Psi_{F} q_{2}, \\
\Psi_{H}=p_{2} \Psi_{H} q_{1},
\end{gathered}
$$

where $p_{i}$ and $q_{i}(i, j=1,2)$ are star algebra projectors defined by

$$
\begin{aligned}
& p_{1}=F c B H, \\
& q_{1}=F B c H, \\
& p_{2}=H c B F, \\
& q_{2}=H B c F .
\end{aligned}
$$

$p_{i}$ and $q_{i}$ are orthogonal projectors since $p_{i}+q_{i}=1$. Projectors with different indexes do not always commute but obey rather nontrivial algebra which is summarized as

$$
\begin{aligned}
& p_{i} p_{j}=p_{i}, \\
& q_{i} q_{j}=q_{j}, \\
& p_{i} q_{j}=0, \\
& q_{i} p_{j}=\epsilon_{i j}\left(p_{j}-p_{i}\right),
\end{aligned}
$$

where $\epsilon_{i j}$ is the antisymmetric tensor. Using these projectors, we can decompose arbitrary string field into projected sectors (we are inspired by a work [28] in which the string field is decomposed by the KMTT projectors). Here we are interested in two kinds of decomposition. One is "D-like" decomposition which is given by

$$
\begin{aligned}
\Psi & =\left(p_{1}+q_{1}\right) \Psi\left(p_{2}+q_{2}\right) \\
& =p_{1} \Psi p_{2}+p_{1} \Psi q_{2}+q_{1} \Psi p_{2}+q_{1} \Psi p_{2} \\
& =\psi_{1}+\psi_{2}+\psi_{3}+\psi_{4},
\end{aligned}
$$

and the other is "gD-like" decomposition

$$
\begin{aligned}
\Psi & =\left(p_{2}+q_{2}\right) \Psi\left(p_{1}+q_{1}\right) \\
& =p_{2} \Psi p_{1}+p_{2} \Psi q_{1}+q_{2} \Psi p_{1}+q_{2} \Psi p_{1} \\
& =\phi_{1}+\phi_{2}+\phi_{3}+\phi_{4} .
\end{aligned}
$$

One can readily find that $\Psi_{\mathrm{D}}=-\Psi_{F}$ and $\Psi_{\mathrm{gD}}=\Psi_{H}$ are components of $\psi_{2}$ and $\phi_{2}$, respectively. Then, the orthogonality between $\Psi_{F}$ and $\Psi_{H}$ is not limited to these solutions but can be extended to the projected sectors:

$$
\psi_{2} \phi_{2}=\phi_{2} \psi_{2}=0
$$

Usually, one may implicitly assume that a nontrivial solution of equation of motion causes background shift for arbitrary string field. However, it is not true if that solution has a kernel. For an illustration, we consider OSFT action around the tachyon vacuum by expanding the fluctuation in terms of (32):

$$
\Psi=\Psi_{F}+\phi_{2}+\eta
$$

where $\eta=\phi_{1}+\phi_{3}+\phi_{4}$. In this case, the string field $\phi_{2}$ is the kernel of the solution $\Psi_{F}$. The OSFT action is expanded as

$$
\begin{aligned}
S[\Psi]= & \frac{1}{2} \operatorname{Tr}\left[\eta Q_{F} \eta\right]+\frac{1}{2} \operatorname{Tr}\left[\phi_{2} Q_{B} \phi_{2}\right]+\operatorname{Tr}\left[\phi_{2} Q_{B} \eta\right] \\
& + \text { (cubic) }+ \text { (const. }) .
\end{aligned}
$$

It is observed that the kinetic operator for $\phi_{2}$ did not shift to $\left(Q_{B}\right)$, while that for $\eta$ is shifted to $Q_{F}$. Therefore, $\phi_{2}$ can be interpreted as a degree of freedom on a "residual" $D$-brane. Equations of motion for the action (35) are

$$
\begin{aligned}
& Q_{B} \phi_{2}+\phi_{2}^{2}+Q_{B} \eta+\phi_{2} \eta+\eta^{2}=0, \\
& Q_{F} \eta+\eta^{2}+Q_{B} \phi_{2}+\phi_{2}^{2}+\phi_{2} \eta=0 .
\end{aligned}
$$

Since $\phi_{2}$ and $\eta$ are projected components, one can project out either of them. By setting $\eta=0$, both of the above equations reduce to the equation of motion for a D-brane

$$
Q_{B} \phi_{2}+\phi_{2}^{2}=0
$$

Thus, tachyon condensation takes place again with a solution $\phi_{2}=\Psi_{H}$. The negative tension of the solution can be interpreted as a result of tachyon condensation in the residual sector. Thus, existence of the residual sector is responsible for the peculiar nature of gD-brane which has negative tension. One can argue whether the residual sector can be removed by gauge fixing. A condition that picks up $\eta$ component from (32) reads

$$
p_{2} \Psi q_{1}=0
$$

This condition looks like a sort of linear $b$ gauge fixing [29] since $p_{2}$ and $q_{1}$ include products of $B$ and $c$. In order to check validity of the condition, one has to show that arbitrary string field can be gauge transformed to satisfy (38), and also that no residual gauge symmetry is left. Proof seems to require detailed and careful evaluation of gauge transformation, so we leave it as a future task.

\section{Multiple Branes}

We next turn to constriction of a solution with arbitrary number of $\mathrm{D}$ - and gD-branes. Basic ingredients of our construction are the modified boundary condition changing (BCC) operators [2]

$$
\begin{aligned}
& \Sigma_{a}=Q_{F}\left(F B \sigma_{a} F\right), \\
& \bar{\Sigma}_{a}=Q_{F}\left(F B \bar{\sigma}_{a} F\right),
\end{aligned}
$$


where $\sigma_{a}$ and $\bar{\sigma}$ are BCC operators associated with certain boundary conditions and $a$ corresponds to Chan-Paton factor which labels D- or gD-brane. By construction, they are $Q_{F}$ exact and therefore vanish when multiplied with it:

$$
Q_{F} \Sigma_{a}=Q_{F} \bar{\Sigma}_{a}=0
$$

They also inherit the algebra of original BCC operators:

$$
\begin{aligned}
& \bar{\Sigma}_{a} \Sigma_{b}=\delta_{a b}, \\
& \Sigma_{a} \bar{\Sigma}_{b}=\text { finite } \times \delta_{a b} .
\end{aligned}
$$

We consider the theory at the tachyon vacuum whose kinetic operator is $Q_{F}$. Then, it is easily understood that, if $\Phi$ is a solution of the equation of motion, $\Sigma_{a} \Phi \bar{\Sigma}_{a}$ is also a solution. Therefore, for given set of solutions $\Phi_{1}, \Phi_{2}, \ldots, \Phi_{N}$, we can construct a set of mutually orthogonal solutions

$$
\Sigma_{1} \Phi_{1} \bar{\Sigma}_{1}, \Sigma_{2} \Phi_{2} \bar{\Sigma}_{2}, \ldots, \Sigma_{N} \Phi_{N} \bar{\Sigma}_{N}
$$

Of course, their sum is also a solution due to the orthogonality of the modified BCC projectors. The sum is conveniently described by vector notation:

$$
\left(\Phi_{1}, \Phi_{2}, \ldots, \Phi_{n}\right)=\sum_{a=1}^{N} \Sigma_{a} \Phi_{a} \bar{\Sigma}_{a}
$$

We can easily construct solutions for multiple $\mathrm{D}$ - or $\mathrm{gD}$ brane, respectively, by

$$
\begin{gathered}
\Psi_{\mathrm{D}}^{(N)}=\underbrace{\left(\Psi_{\mathrm{D}}, \Psi_{\mathrm{D}}, \ldots, \Psi_{\mathrm{D}}\right)}_{N}, \\
\Psi_{\mathrm{gD}}^{(N)}=\underbrace{\left(\Psi_{\mathrm{gD}}, \Psi_{\mathrm{gD}}, \ldots, \Psi_{\mathrm{gD}}\right)}_{N},
\end{gathered}
$$

where $\Psi_{\mathrm{D}}$ and $\Psi_{\mathrm{gD}}$ are solutions defined by (14) and (17), respectively. Due to the orthogonality, gauge invariant observables split into pieces for each component. For example, classical action for D-branes is evaluated as

$$
\begin{aligned}
S_{F}\left[\Psi_{\mathrm{D}}^{(N)}\right] & =N S_{F}\left[\Psi_{\mathrm{D}}\right] \\
& =N .
\end{aligned}
$$

Similar evaluation for gD-branes derives the value $-N$. Then the cancellation between tensions can be confirmed as follows. First, we construct a pair of $N$ D-branes and $N$ gDbranes. Note that $\Psi_{\mathrm{D}}^{(N)}+\Psi_{\mathrm{gD}}^{(N)}$ again becomes a solution since

$$
\Psi_{\mathrm{D}+\mathrm{gD}}^{(N)}=\Psi_{\mathrm{D}}^{(N)}+\Psi_{\mathrm{gD}}^{(N)}=\underbrace{\left(\Psi_{\mathrm{D}+\mathrm{gD}}, \Psi_{\mathrm{D}+\mathrm{gD}}, \ldots, \Psi_{\mathrm{D}+\mathrm{gD}}\right)}_{N},
$$

where $\Psi_{\mathrm{D}+\mathrm{gD}}=\Psi_{\mathrm{D}}+\Psi_{\mathrm{gD}}$ is a D-gD pair defined by (16). Then, the tension cancels as

$$
S_{F}\left[\Psi_{\mathrm{D}+\mathrm{gD}}^{(N)}\right]=S_{F}\left[\Psi_{\mathrm{D}}^{(N)}\right]+S_{F}\left[\Psi_{\mathrm{gD}}^{(N)}\right]=+N-N=0 .
$$

Similar cancellations also hold for other observables.
We next ask whether the cancellation of cohomology occurs between $\mathrm{D}$ - and gD-branes. To see this, let us consider the kinetic operator around $\Psi_{\mathrm{D}+\mathrm{gD}}^{(\mathrm{N})}$. It is given by

$$
Q_{\mathrm{D}+\mathrm{gD}}^{(N)} \Psi=Q_{F} \Psi+\left\{\Psi_{\mathrm{D}+\mathrm{gD}}^{(N)}, \Psi\right\} .
$$

Right hand side of (49) does not seem to correspond to any known operator with empty cohomology. However, it can be shown that this operator splits into $n$ copies of kinetic operator with empty cohomology for particular choice of $\Psi$. Such choice of a string field is namely given by a vector of same kind as (44) or (45):

$$
\Psi^{(N)}=\underbrace{\left(\Psi_{1}, \Psi_{2}, \ldots, \Psi_{N}\right)}_{N} .
$$

Note that this choice corresponds to a subset of projected string fields considered in [28]. Then, with (40) and (41), it is straightforward to show that

$$
Q_{\mathrm{D}+\mathrm{gD}}^{(N)} \Psi^{(N)}=\underbrace{\left(Q_{\mathrm{D}+\mathrm{gD}} \Psi_{1}, Q_{\mathrm{D}+\mathrm{gD}} \Psi_{2}, \ldots, Q_{\mathrm{D}+\mathrm{gD}} \Psi_{N}\right)}_{N},
$$

where $Q_{D+g D}$ is a kinetic operator for a $\mathrm{D}-\mathrm{gD}$ pair given by (26). Thus, the kinetic operator around $N$ D-gD pair splits into $Q_{\mathrm{D}+\mathrm{gD}}$ which has no cohomology. Therefore, cancellation between cohomologies holds for the projected string field (50).

A solution with different numbers of D- and gD-branes can be constructed similarly. For example, consider

$$
\begin{aligned}
& \Psi_{\mathrm{D}}^{(N+K)}=(\underbrace{\Psi_{\mathrm{D}}, \Psi_{\mathrm{D}}, \ldots, \Psi_{\mathrm{D}}}_{N}, \underbrace{\Psi_{\mathrm{D}}, \Psi_{\mathrm{D}}, \ldots, \Psi_{\mathrm{D}}}_{K}, \\
& \underbrace{0,0, \ldots, 0}_{M}), \\
& \Psi_{\mathrm{gD}}^{(K+M)}=(\underbrace{0,0, \ldots, 0}_{N}, \underbrace{\Psi_{\mathrm{gD}}, \Psi_{\mathrm{gD}}, \ldots, \Psi_{\mathrm{gD}}}_{K}, \\
& \underbrace{\Psi_{\mathrm{gD}}, \Psi_{\mathrm{gD}}, \ldots, \Psi_{\mathrm{gD}}}_{M}) .
\end{aligned}
$$

A sum $\Psi_{\mathrm{D}}^{(N+K)}+\Psi_{\mathrm{gD}}^{(K+M)}$ is a solution that represents $N+K$ D-branes and $K+M$ gD-branes. Since $\Psi_{\mathrm{D}}+\Psi_{\mathrm{gD}}=\Psi_{\mathrm{D}+\mathrm{gD}}$, the sum is written as

$$
\begin{gathered}
\Psi^{(N, K, M)}=\Psi_{\mathrm{D}}^{(N+K)}+\Psi_{\mathrm{gD}}^{(K+M)}=(\underbrace{\Psi_{\mathrm{D}}, \Psi_{\mathrm{D}}, \ldots, \Psi_{\mathrm{D}}}_{N}, \\
\underbrace{\Psi_{\mathrm{D}+\mathrm{gD}}, \Psi_{\mathrm{D}+\mathrm{gD}}, \ldots, \Psi_{\mathrm{D}+\mathrm{gD}}}_{K}, \underbrace{\Psi_{\mathrm{gD}}, \Psi_{\mathrm{gD}}, \ldots, \Psi_{\mathrm{gD}}}_{M}) .
\end{gathered}
$$

The value of the classical action is given by $N-M$ as expected. However, the cohomology cancels only in $k$ slots in the 
middle of the vector (53). This can be shown as follows. We introduce a shorthand notation

$$
\Phi=\left(\Phi^{(N)}, \Phi^{(K)}, \Phi^{(M)}\right)
$$

where three components stand for vectors in each $N, K$, and $M$ slot. Then, kinetic operator around $\Psi^{(N, K, M)}$ is evaluated as

$$
\begin{aligned}
Q_{F} & \Phi+\left\{\Psi^{(N, K, M)}, \Phi\right\} \\
& =\left(Q_{B} \Phi^{(N)}, Q_{\mathrm{D}+\mathrm{gD}} \Phi^{(K)}, Q_{\mathrm{gD}} \Phi^{(M)}\right)
\end{aligned}
$$

where $Q_{\mathrm{gD}}=Q_{F}+\left\{\Psi_{\mathrm{gD}}, *\right\}$ is a kinetic operator for a $\mathrm{gD}$ brane. It is obvious that only $Q_{\mathrm{D}+\mathrm{gD}}$ has vanishing cohomology; therefore, cancellation occurs only in the middle $K$ slots. First $N$ and last $M$ slots have no chance to cancel since their Chan-Paton factors do not overlap.

We can further extend above system by introducing offdiagonal Chan-Paton factors of $\Phi$ following the method of [2, 28]. We introduce KMTT projectors $P_{k}=\Sigma_{k} \bar{\Sigma}_{k}$ and arrange them as

$$
\left\{P_{0}, P_{a}, P_{\alpha}, P_{A}\right\}
$$

where $a, \alpha$, and $A$ are assigned to three slots of indexes in (53), and $P_{0}=1-\sum_{k=1}^{N+K+M} \sum_{k} \bar{\Sigma}_{k}$ is a complementary projector. These labels are identified with Chan-Paton factors for each brane; $0, a, \alpha$, and $A$ are assigned to a tachyon vacuum, $N$ Dbranes, $K$ tachyon vacuum, and $M$ gD-branes, respectively. This assignment comes from the fact that a component of string field in each sector obeys appropriate kinetic term in the OSFT action expanded around (53). For example, as for $\Phi_{a b}=P_{a} \Psi P_{b}$,

$$
Q_{F} \Phi_{a b}+\left\{\Psi^{(N, K, M)}, \Phi_{a b}\right\}=Q_{B} \Phi_{a b}
$$

holds. The component $\Phi_{a b}$ corresponds to open string field between D-brane $a$ and $b$ since $Q_{B}$ is a kinetic operator for a D-brane. A component with mixed indexes, like $\Phi_{a A}$, is interpreted as a string field which connects different kinds of branes.

\section{Conclusions}

In this paper, we have studied ghost D-branes in the context of open string field theory. First, we constructed the classical solutions for the D-gD system with a help of the MNT solutions. We have confirmed cancellation of gauge invariant observables for the D-gD pair. It has been shown that the BRST cohomology of a D-gD pair cancels. Next we have extended previous result to a system with arbitrary numbers of $\mathrm{D}$ - and gD-branes. We have constructed corresponding solution using modified BCC operators [2]. It has shown that cancellation of cohomology holds for branes with common Chan-Paton factors.

We would like to answer the two questions asked in Introduction. Fist question asks whether gD-branes are physical objects. While we did not find conclusive answer to this, we found that gD-branes belong to a sector of open string field which is not affected by the shift of background. As mentioned in Section 2, we can ask whether such sector is gauge away. If so, gD-branes turn out to be unphysical objects and we do not need to worry about negative tension. On the other hand, severe problem will remain if the $\mathrm{gD}$ sector cannot be removed. Multiple gD-branes constructed in Section 3 make the spectrum of OSFT unbounded below. Therefore, it remains important to give conclusive answer to this question.

Second question asks about difference between the original picture of [5] and OSFT prescription. We have confirmed that the BRST cohomology cancels between $\mathrm{D}$ - and $\mathrm{gD}$ branes. Therefore, quantum fluctuations around a $\mathrm{D}-\mathrm{gD}$ pair cancel expectedly. However, unlike the original picture, the cancellation cannot be extended to the whole partition function. This discrepancy leads to peculiar phenomenon in OSFT. Let us consider multiple D-gD system. The kinetic operator of a D-gD pair is given by $O_{\mathrm{D}+\mathrm{gD}}=Q_{H}$ which is equivalent to that of tachyon vacuum. One can consider an effective action for remaining branes by integrating out string fields for cancelled D-gD pairs. As has been conjectured earlier [30-33], such integration will leave closed string amplitudes with no boundaries, that is, closed string tadpoles. Therefore, infinitely many D-gD pair implies infinitely many tadpoles. Although this seems rather pathological, useful applications and implications will be expected.

Finally, we present rather positive perspective of our result. Since a D-gD pair is equivalent to the tachyon (or the closed string) vacuum, we can say that the closed string vacuum is described by two vacua in different BCFTs, that is, one for $\mathrm{D}$-brane and the other for $\mathrm{gD}$-brane. It is expected that an extension to excited states of closed string will lead to brand-new formulation of closed string theory in terms of open string. Even if gD-brane is unphysical, it will play a role of auxiliary degrees of freedom which is useful for such formulation.

To push this program forward, we should identify BCFT for gD-brane. Naively, it is expected that a gD-brane carries same boundary condition as that of a D-brane since boundary states only differ in their sign. In order to identify gD-brane BCFT in OSFT context, one has to derive cohomology of gD-brane. However, direct identification of it seems to be not straightforward, as some of our attempts indicate. For example, formal homotopy operator of the gD-brane kinetic operator

$$
Q_{F+H}=Q_{B}+\left\{\Psi_{F}+\Psi_{H}, *\right\}
$$

seems to vanish at least within $K B c$ algebra. This is not similar to the D-brane cohomology whose $B / K$ is a formal homotopy operator. Another but related attempt is finding left and right transformations $[19,34]$ which connect D- and gD-branes. This had been already derived by MNT [17] as

$$
U_{L}=M K \frac{F}{1-F^{2}} c B \frac{1-F^{2}}{F}=M K J c B J^{-1},
$$

where $J=F /\left(1-F^{2}\right)$. The latter piece $J c B J^{-1}$ is a star algebra projector and therefore has a nontrivial kernel regardless of 
potential singularity due to $K=0$ poles of $M$ and $F^{-1}$. Cohomologies of $\mathrm{D}$ - and $\mathrm{gD}$-branes may be related in projected space of string fields obtained by excluding this kernel. To establish this, it is necessary to confirm whether the projected space is suitable to describe $\mathrm{D}$ - or gD-brane BCFT.

\section{Conflicts of Interest}

The author declares that there are no conflicts of interest regarding the publication of this paper.

\section{Acknowledgments}

The author would like to thank organizers of the conference "Progress in Quantum Field Theory and String Theory II" at Osaka City University for an opportunity of poster presentation. Discussions with participants of the conference were quite useful to complete our research. In particular, the author would like to thank Masako Asano, Hiroshi Itoyama, Isao Kishimoto, and Keisuke Ohashi for valuable discussions. A support from JSPS/RFBR bilateral project "Faces of Matrix Models in Quantum Field Theory and Statistical Mechanics" is gratefully appreciated.

\section{References}

[1] E. Witten, "Noncommutative geometry and string field theory," Nuclear Physics. B. Theoretical, Phenomenological, and Experimental High Energy Physics. Quantum Field Theory and Statistical Systems, vol. 268, no. 2, pp. 253-294, 1986.

[2] T. Erler and C. Maccaferri, "String field theory solution for any open string background," Journal of High Energy Physics, vol. 2014, no. 10, article 29, 2014.

[3] J. L. Cardy, Boundary Conformal Field Theory, arXiv:hepth/0411189.

[4] T. Kojita, C. Maccaferri, T. Masuda, and M. Schnabl, Topological Defects in Open String Field Theory, arXiv:1612.01997.

[5] T. Okuda and T. Takayanagi, "Ghost D-branes," Journal of High Energy Physics. A SISSA Journal, no. 3, 062, 39 pages, 2006.

[6] S. Terashima, "Ghost D-brane, supersymmetry and matrix model," Journal of High Energy Physics. A SISSA Journal, no. 5, 067, 11 pages, 2006.

[7] N. Evans, T. R. Morris, and O. J. Rosten, "Gauge invariant regularization in the AdS/CFT correspondence and ghost Dbranes," Physics Letters. B. Particle Physics, Nuclear Physics and Cosmology, vol. 635, no. 2-3, pp. 148-150, 2006.

[8] C. Vafa, BranE/Anti-Brane Systems and $U(N-M)$ Supergroup, arXiv:hep-th/0101218.

[9] S. E. Parkhomenko, "BRST construction of D-branes in SU(2) WZW model," Nuclear Physics. B. Theoretical, Phenomenological, and Experimental High Energy Physics. Quantum Field Theory and Statistical Systems, vol. 617, no. 1-3, pp. 198-214, 2001.

[10] S. E. Parkhomenko, "Free field construction of D-branes in $N=2$ superconformal minimal models," Nuclear Physics. B. Theoretical, Phenomenological, and Experimental High Energy Physics. Quantum Field Theory and Statistical Systems, vol. 671, no. 1-3, pp. 325-342, 2003.

[11] S. E. Parkhomenko, "Free-field approach to D-branes in Gepner models," Nuclear Physics. B. Theoretical, Phenomenological, and
Experimental High Energy Physics. Quantum Field Theory and Statistical Systems, vol. 731, no. 3, pp. 360-388, 2005.

[12] S. E. Parkhomenko, "Free field construction of D-branes in rational models of CFT and Gepner models," SIGMA, vol. 4, article 025, 2008.

[13] R. Dijkgraaf, B. Heidenreich, P. Jefferson, and C. Vafa, Negative Branes, Supergroups and the Signature of Spacetime, arXiv:1603.05665.

[14] M. Murata and M. Schnabl, "Multibrane solutions in open string field theory," Journal of High Energy Physics, vol. 2012, no. 7, article 063, 2012.

[15] H. Hata and T. Kojita, "Winding number in string field theory," Journal of High Energy Physics, vol. 2012, no. 1, article 088, 2012.

[16] H. Hata and T. Kojita, "Singularities in K-space and multi-brane solutions in cubic string field theory," Journal of High Energy Physics, vol. 2013, no. 2, pp. 1-18, 2013.

[17] T. Masuda, T. Noumi, and D. Takahashi, "Constraints on a class of classical solutions in open string field theory," Journal of High Energy Physics, vol. 2012, no. 10, article no. 113, 2012.

[18] T. Erler, "The identity string field and the sliver frame level expansion," Journal of High Energy Physics, vol. 2012, no. 11, article no. 150, 2012.

[19] T. Erler and C. Maccaferri, "Connecting solutions in open string field theory with singular gauge transformations," Journal of High Energy Physics, vol. 2012, no. 4, article no. 107, 2012.

[20] Y. Baba, N. Ishibashi, and K. Murakami, "D-branes and closed string field theory," Journal of High Energy Physics. A SISSA Journal, no. 5, article 029, 2006.

[21] Y. Baba, N. Ishibashi, and K. Murakami, "D-brane states and disk amplitudes in OSp invariant closed string field theory," Journal of High Energy Physics. A SISSA Journal, no. 10, article 008, 2007.

[22] Y. Okawa, "Comments on Schnabl's analytic solution for tachyon condensation in Witten's open string field theory," Journal of High Energy Physics. A SISSA Journal, no. 4, article 055, 2006.

[23] M. Kiermaier, Y. Okawa, and B. Zwiebach, The Boundary State from Open String Fields, arXiv:0810.1737.

[24] I. Ellwood, "The closed string tadpole in open string field theory," Journal of High Energy Physics. A SISSA Journal, no. 8, article 063, 2008.

[25] T. Kawano, I. Kishimoto, and T. Takahashi, "Gauge invariant overlaps for classical solutions in open string field theory," Nuclear Physics. B. Theoretical, Phenomenological, and Experimental High Energy Physics. Quantum Field Theory and Statistical Systems, vol. 803, no. 1-2, pp. 135-165, 2008.

[26] M. Kudrna, C. Maccaferri, and M. Schnabl, "Boundary state from Ellwood invariants," Journal of High Energy Physics, vol. 2013, no. 7, article no. 033, 2013.

[27] I. Ellwood and M. Schnabl, "Proof of vanishing cohomology at the tachyon vacuum," Journal of High Energy Physics. A SISSA Journal, no. 2, article 096, 2007.

[28] I. Kishimoto, T. Masuda, T. Takahashi, and S. Takemoto, "Open string fields as matrices," PTEP. Progress of Theoretical and Experimental Physics, no. 3, article 033B05, 2015.

[29] M. Kiermaier, A. Sen, and B. Zwiebach, "Linear b-gauges for open string fields," Journal of High Energy Physics. A SISSA Journal, no. 3, article 050, 2008.

[30] D. Gaiotto, L. Rastelli, A. Sen, and B. Zwiebach, "Ghost structure and closed strings in vacuum string field theory," Advances in 
Theoretical and Mathematical Physics, vol. 6, no. 3, pp. 403-456, 2002.

[31] N. Drukker, "Closed string amplitudes from gauge fixed string field theory," Physical Review D: Particles, Fields, Gravitation and Cosmology, vol. 67, no. 12, article 126004, 2003.

[32] N. Drukker, "On different actions for the vacuum of bosonic string field theory," Journal of High Energy Physics. A SISSA Journal, no. 8, article 017, 2003.

[33] T. Takahashi and S. Zeze, "Closed string amplitudes in open string field theory," Journal of High Energy Physics. A SISSA Journal, no. 8, article 020, 2003.

[34] T. Erler and C. Maccaferri, "The phantom term in open string field theory," Journal of High Energy Physics, no. 6, article 084, 2012. 

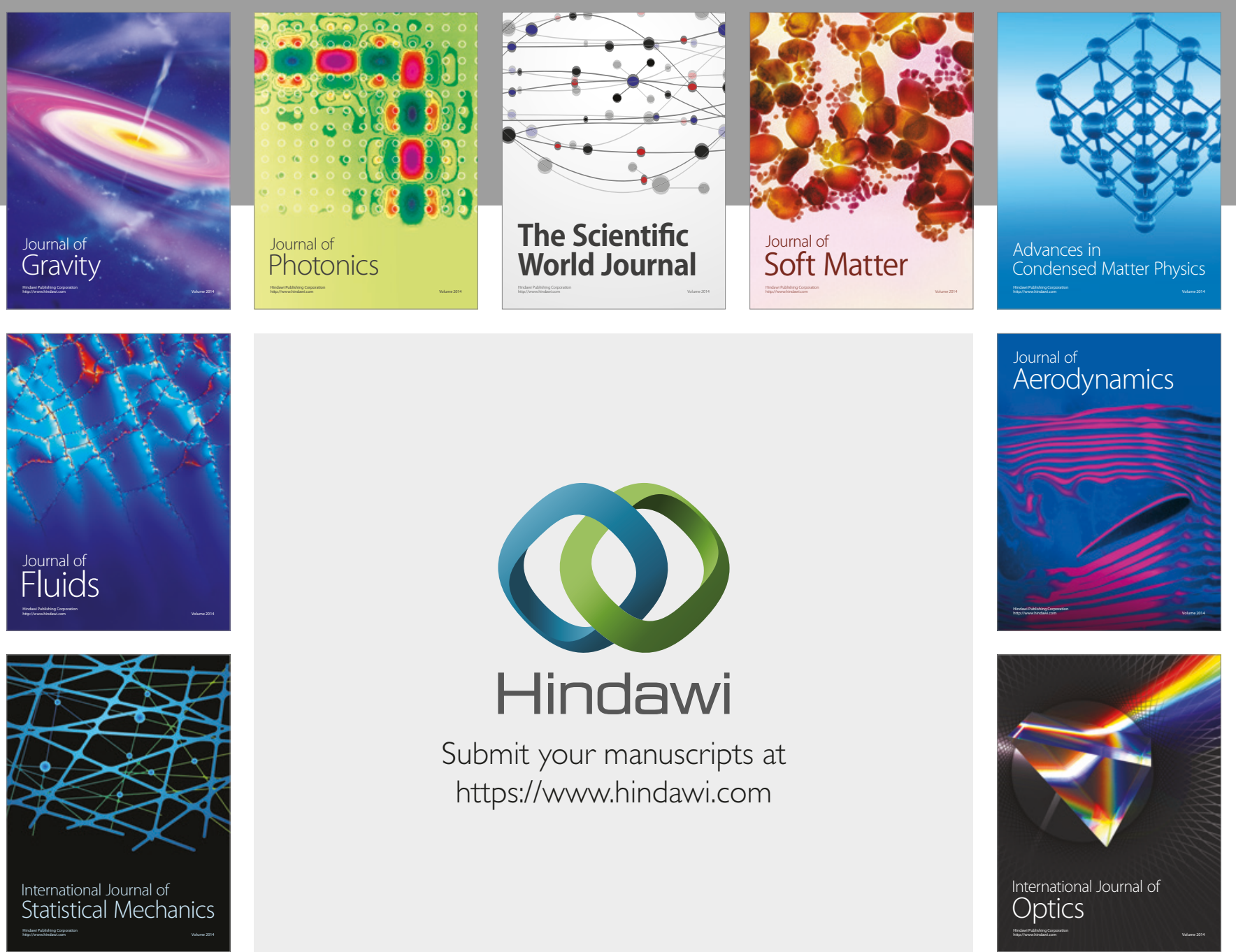

Submit your manuscripts at

https://www.hindawi.com
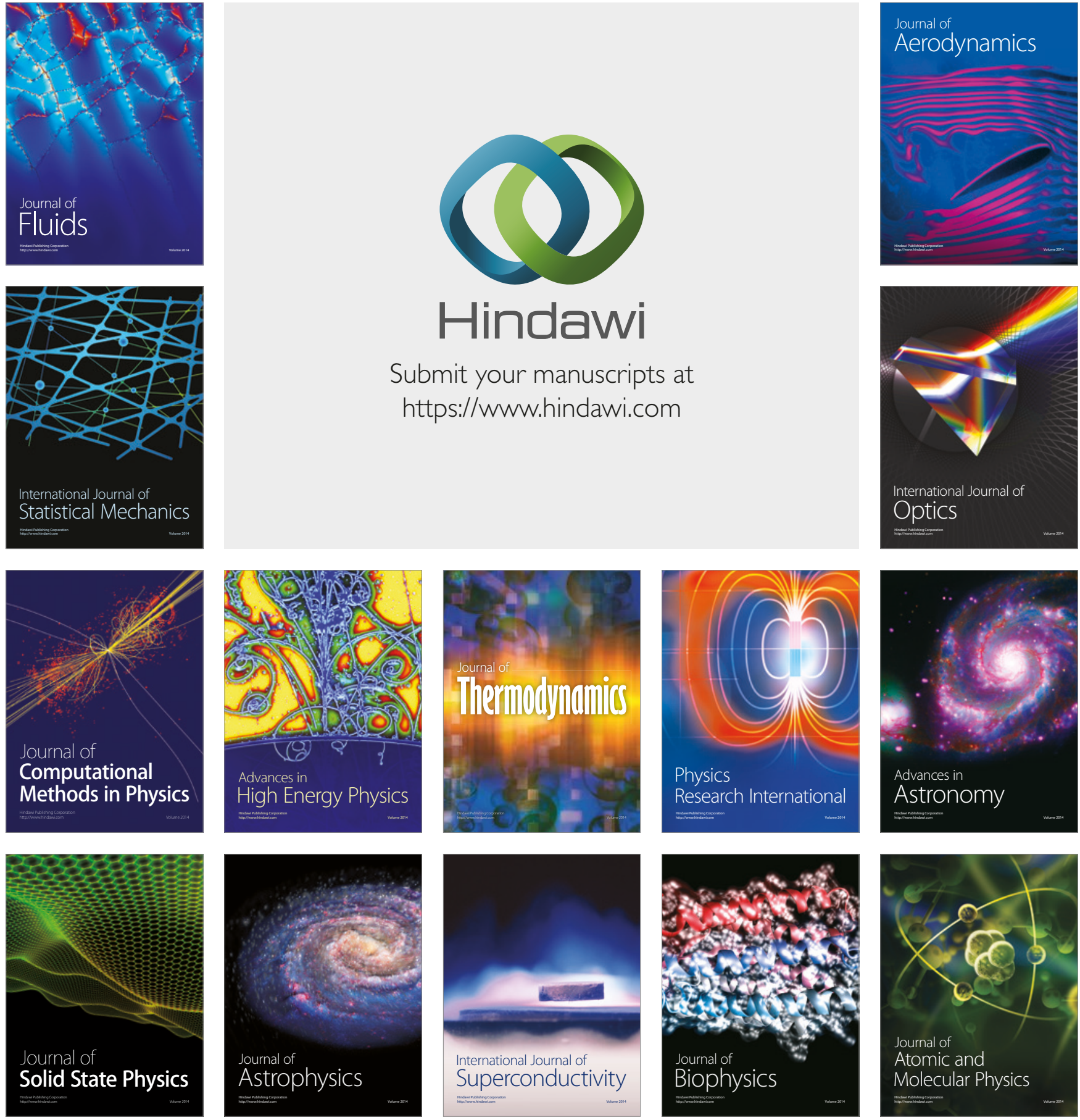